\title{
Osificación del ligamento estilohioideo como causa de dolor neuropático grave: enfoque del cirujano de cabeza y cuello
}

\author{
Ossification of the stylohyoid ligament as cause of severe neuropathic pain: \\ approach by the head and neck surgeon \\ Andrey Moreno ${ }^{1}$, Sebastián Sánchez² ${ }^{2}$ Paula Flórez${ }^{3}$, Mauricio Pedraza ${ }^{2}$, Luis Felipe Cabrera ${ }^{4}$ \\ Médico, especialista en Cirugía General, Departamento de Cirugía de Cabeza y Cuello, Hospital Universitario de La Samaritana, \\ Bogotá, D.C., Colombia \\ 2 Médico, especialista en Cirugía General, Universidad El Bosque, Bogotá, D.C., Colombia \\ 3 Médico, residente de Cirugía General, Departamento de Cirugía,, Universidad de La Sabana, Chía, Colombia \\ 4 Médico, especialista en Cirugía General, Universidad El Bosque, Los Cobos Medical Center, Bogotá, D.C., Colombia
}

\section{Resumen}

Introducción. La osificación de la cadena estilohioidea es una entidad poco frecuente que se caracteriza por dolor neuropático importante en la región facial y la cervical, secundario a la compresión mecánica de las estructuras vasculares y nerviosas del cuello. Tradicionalmente, se trata de una enfermedad de manejo por el otorrinolaringólogo, aunque en algunos casos es necesaria la intervención del cirujano de cabeza y cuello. Es por ello que, en el presente artículo, se presenta un reporte de caso y se hace una revisión de la literatura científica.

Caso clínico. Se presenta el caso de una paciente de 65 años de edad que consultó por dolor cervical y facial serio, secundario a la osificación del complejo estilohioideo, con necesidad de manejo quirúrgico. Por medio de una cervicotomía, se extrajo la pieza osificada, sin complicaciones perioperatorias y resultados favorables.

Discusión. La osificación del complejo estilohioideo es una situación poco frecuente, con un cuadro clínico inespecífico y mecanismos fisiopatológicos desconocidos, que puede llevar a circunstancias potencialmente mortales. Para llegar a su diagnóstico, se debe tener un alto grado de sospecha y se confirma con una tomografía de cuello con reconstrucción tridimensional. Su tratamiento es quirúrgico, aunque también se ha descrito el manejo médico. En la literatura médica, no existen estudios que comparen los métodos diagnósticos y terapéuticos para esta entidad, por lo cual, hasta el día de hoy, existen grandes controversias al respecto. Esto hace necesario que se lleven a cabo nuevas investigaciones en este campo.

Palabras clave: síndrome de Eagle; hueso hioides; osificación del ligamento; dolor facial; dolor de cuello; cirugía.

Fecha de recibido: 22/06/2018 - Fecha aceptación: 19/09/2018

Correspondencia: Andrey Moreno, MD, Calle 127 \# 20 - 16 Consultorio 214 Edifico Pluss 127, Bogotá, D.C., Colombia,

Teléfono: (+57) (311) 287-9443

Correo electrónico: andreymorenotorres@gmail.com

Citar como: Moreno A, Sánchez S, Flórez P, Pedraza M, Cabrera LF. Osificación del ligamento estilohioideo como causa de dolor neuropático grave: enfoque del cirujano de cabeza y cuello. Rev Colomb Cir. 2019;34:185-9. https://doi.org/10.30944/20117582.113

Este es un artículo de acceso abierto bajo una Licencia Creative Commons - BY-NC-ND https://creativecommons.org/licenses/by-nc-nd/4.0/deed.es 


\begin{abstract}
Introduction: Stylohyoid chain ossification is a rare entity characterized by severe neuropathic pain in the facial region secondary to mechanical compression of neurovascular structures of the neck. Traditionally it is a pathology that requires otorhinolaryngological management, although in some cases the intervention of the head and neck surgeon is necessary. That is why in this article we present the management of a patient with this pathology.

Clinical case: Our case is a 65-year-old woman who consulted for severe neck and facial pain secondary to the ossification of the stylohyoid complex and the requirement for surgical management. By means of cervicotomy the ossified piece is extracted, without any perioperative complication and favorable results.

Discussion: Stylohyoid chain ossification is a rare pathology, with a nonspecific clinical picture which can lead to potentially fatal circumstances. To reach the diagnosis, the surgeon is required to have a high degree of suspicion and confirmation with a neck CT scan with $3 \mathrm{D}$ reconstruction. The treatment for this entity is usually surgical, although medical management has also been described.

There are no studies reported in the medical literature comparing the diagnostic and therapeutic methods for this entity, so that until today, major controversies in this regard still remains. It becomes necessary new research in this field.
\end{abstract}

Key words: Eagle syndrome; hyoid bone; ossification of ligament; facial pain; neck pain; surgery.

\section{Introducción}

La osificación de la cadena estilohioidea es una entidad poco frecuente que se caracteriza por un dolor neuropático importante en la región facial y la cervical, secundario a la compresión mecánica de las estructuras vasculares y nerviosas del cuello. Tradicionalmente, se trata de una entidad de manejo por el otorrinolaringólogo, aunque, en algunos casos, se hace necesaria la intervención del cirujano de cabeza y cuello; por ello, es de carácter imperativo que el médico general y el cirujano general conozcan esta entidad para poder diagnosticarla y darle un manejo integral ${ }^{\mathrm{I}-4}$.

Por lo anterior, en el presente artículo se presenta nuestra experiencia en el manejo de esta entidad y se hace una revisión crítica de la literatura científica.

\section{Caso clínico}

Se trata de una paciente de sexo femenino de 65 años de edad, con dolor de tipo punzada de intensidad moderada a seria en la hemicara y en el hemicuello izquierdos, de seis meses de evolución, asociado con disfagia y odinofagia.

Fue valorada de manera ambulatoria por el Servicio de Otorrinolaringología, donde consideraron que la paciente cursaba con un síndrome de Eagle por los hallazgos clínicos y tomográficos, por lo cual fue sometida a una resección de la apófisis estiloides izquierda, sin que se obtuviera mejoría de la sintomatología.

Ante dicha situación, descartaron la elongación de la apófisis estiloides izquierda como agente causal del dolor y decidieron solicitar valoración por el Servicio de Cirugía de Cabeza y Cuello.

Como antecedentes relevantes refirió amigdalectomía palatina bilateral.

En el examen físico se encontró: frecuencia cardiaca de 83 latidos por minuto, tensión arterial de $124 / 78 \mathrm{~mm} \mathrm{Hg}$, frecuencia respiratoria de $\mathrm{I} 7$ por minuto y temperatura de $36,5^{\circ} \mathrm{C}$. El cuello era móvil y doloroso a la palpación en la región submandibular izquierda, en donde se palpaba una masa dura de $2 \times 2 \mathrm{~cm}$, fija y adherida a los planos profundos; no hubo otros hallazgos relevantes.

En una nueva tomografía computadorizada de cuello con reconstrucción tridimensional, se observó una apófisis accesoria en el lado izquierdo del hioides, así como elongación de la apófisis estiloides derecha y ausencia posquirúrgica de la izquierda. Sin embargo, existía la posibilidad de que el asta accesoria del hioides correspondiera a 
la osificación caudal del ligamento estilohioideo (figura I).

Dada la evolución clínica y la persistencia de la sintomatología, se decidió intervenirla quirúrgicamente para resecar el ligamento estilohioideo osificado.

Mediante una cervicotomía transversa submandibular izquierda, con disección del músculo platisma y de la aponeurosis superficial, se accedió al hioides (figura 2). Al desinsertar el tendón del músculo digástrico, se evidenció una estructura alargada calcificada unida al hioides, por lo que se consideró una duplicación del asta lateral izquierda frente a una calcificación del ligamento estilohioideo. Se procedió a sujetar dicha estructura con una pinza gubia y resecarla por completo (figura 3). Finalmente, se continuó con el cierre por planos de los músculos y la piel.

El tiempo quirúrgico fue de 30 minutos, hubo mínimo sangrado y no se presentaron complicaciones perioperatorias. En el posoperatorio, la paciente presentó mejoría significativa del dolor
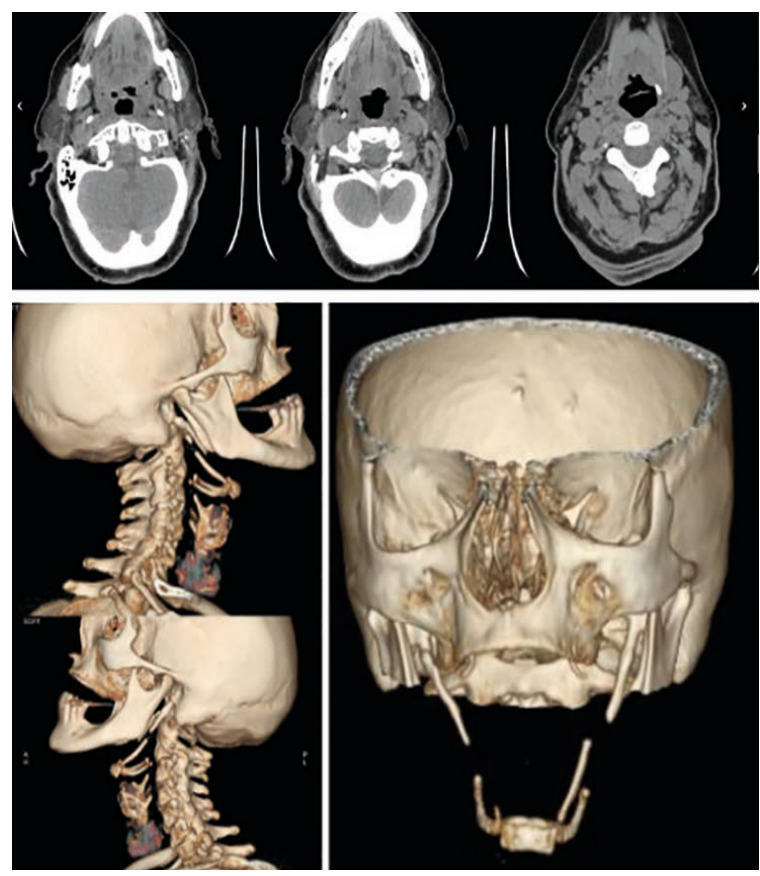

Figura 1. Tomografía computarizada de cuello con reconstrucción tridimensional en donde se observa la osificación del complejo estilohioideo. y toleró la vía oral; se le dio de alta al segundo día del procedimiento.

\section{Discusión}

En 1937, Watt W. Eagle describió por primera vez la calcificación del complejo estilohioideo, con una elongación de la apófisis estiloides del temporal como agente causante de dolor cérvicofacial grave ${ }^{3-10}$.

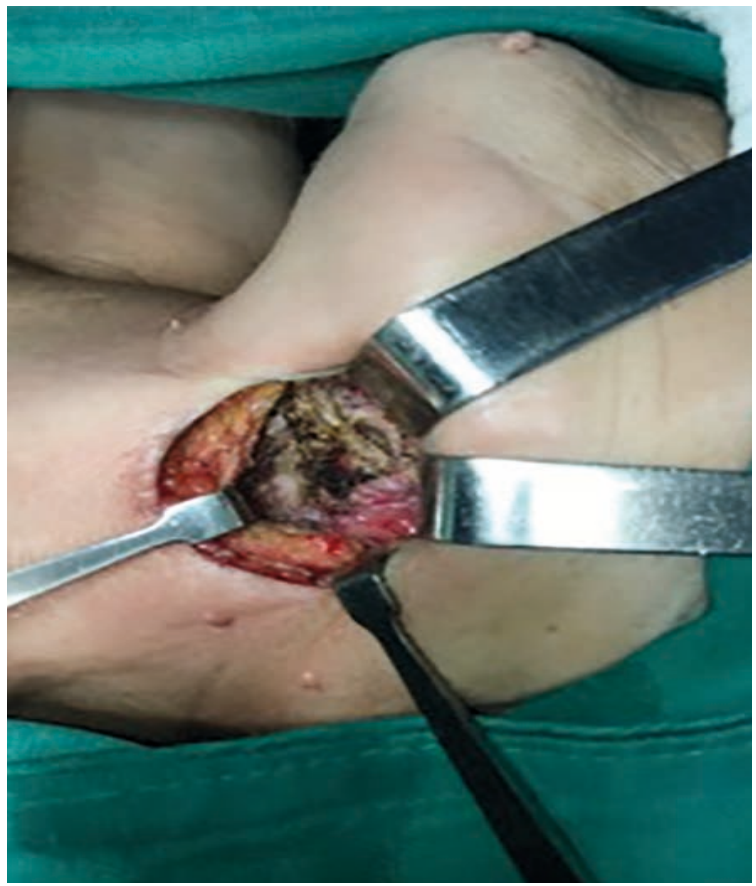

Figura 2. Cervicotomía en donde se observa la osificación del complejo estilohioideo a extraer.

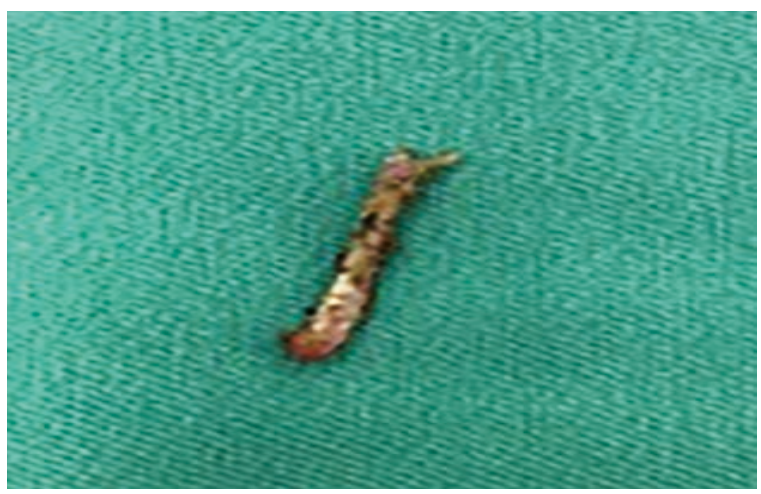

Figura 3. Pieza osificada extraída 
La cadena estilohioidea está compuesta por tres estructuras: la apófisis estiloides del temporal, el hueso hioides y el ligamento estilohioideo ${ }^{1,5}$. Una apófisis estiloides con una longitud superior a $30 \mathrm{~mm}$, tiene una gran probabilidad de causar sintomatología, sobre todo, cuando se asocia con calcificación caudal del ligamento estilohioideo, como en el presente caso ${ }^{6,7}$.

Según los diferentes informes publicados, la osificación de la cadena estilohioidea tiene una incidencia que varía entre 0,16 y i8 \%; sin embargo, tan solo 4 a Io \% de estos pacientes presentan sintomatología ${ }^{3-5}$. Esta condición es más frecuente en mujeres y, usualmente, se diagnostica después de los 30 años ${ }^{1,4,6,7}$. No obstante, existen reportes como el de Gárriz-Luis, et al., de $2017^{9}$, de pacientes menores de Io años que inicialmente empezaron a presentar dolor facial grave, secundario a un síndrome estilohioideo.

Los mecanismos fisiopatológicos aún no están bien dilucidados y la etiología se desconoce. Sin embargo, se han planteado teorías que proponen como agentes causales de la entidad a la organogénesis inadecuada, la herencia, el trauma, la menopausia, el envejecimiento o la degeneración del tejido, ${ }^{1,45,9,9,10}$. Por sus características tisulares, el ligamento estilohioideo tiene una mayor probabilidad de presentar osificación, la cual se ha relacionado con la manipulación quirúrgica previa del cuello, como en el presente caso ${ }^{1,7}$.

Aunque es poco frecuente, este tipo de alteraciones anatómicas se ha asociado con la muer-

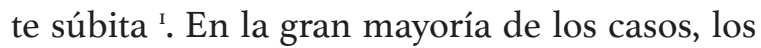
pacientes son asintomáticos, aunque, en ciertas circunstancias, se puede presentar una amplia gama de síntomas, que varían según las estructuras anatómicas comprometidas ${ }^{2}$. El síntoma cardinal en esta entidad es la denominada 'estilalgia', la cual consiste en dolor facial y cervical unilateral, disfagia y disfonía, como en el presente caso.

También, puede presentarse afectación de los nervios trigémino, glosofaríngeo, facial y vago, así como compresión de las arterias carótidas, que lleva a odinofagia, cefalea, dolor de hombro, mareo, acúfenos, sensación de cuerpo extraño o masa, déficit neurológico o alteración del estado de conciencia ${ }^{3,5-7}$.

Algunos autores aseguran que este tipo de malformaciones puede provocar que el paciente acuda al servicio de urgencias, como lo reportaron Smoot, et al., en un paciente que presentó un cuadro clínico de disección carotídea y compromiso neurológico secundario a una osificación del complejo estilohioideo ${ }^{3}$.

El hallazgo patognomónico de esta entidad es una apófisis estiloides palpable a nivel de los pilares palatinos; sin embargo, esta exploración se hace con poca frecuencia durante el examen físico ${ }^{2}$. En la mayoría de los casos, el diagnóstico se hace con ayuda de estudios imagenológicos (radiografía simple, tomografía computadorizada de cuello y resonancia magnética) o durante las necropsias. La tomografía computadorizada de cuello con reconstrucción tridimensional es el estudio de elección para llegar al diagnóstico, como en el presente caso ${ }^{2,4,9,11}$.

Existen pocas publicaciones sobre este tema y no sabemos de ninguna guía para el diagnóstico o el tratamiento de esta entidad. Aunque se ha descrito el manejo médico con calor, medicamentos antiinflamatorios y analgésicos, el tratamiento de esta entidad es quirúrgico y consiste en la resección del componente osificado, con el objetivo de descomprimir las estructuras vasculares y nerviosas del cuello ${ }^{3}$.

El abordaje para la resección puede ser a través de la cavidad oral o extraoral, como en el presente caso que se hizo por medio de una cervicotomía. Algunos autores argumentan que debe preferirse el abordaje por cervicotomía, pues permite una mejor visualización de las estructuras del cuello, a pesar de relacionarse con tiempos quirúrgicos más prolongados y peores resultados estéticos ${ }^{7, \mathrm{II}}$; sin embargo, no existen estudios en los que se comparen estos dos tipos de abordaje.

Consideramos que para el síndrome de Eagle, el abordaje ideal es el transoral, pues el acceso a las apófisis estiloides alargadas es fácil a través de la musculatura faríngea; no obstante, cuando se trata de osificación del ligamento estilohioideo en su tercio distal, como en el presente caso, de- 
bería preferirse la cervicotomía, con una incisión no mayor de $3 \mathrm{~cm}$.

\section{Conclusión}

La osificación del complejo estilohioideo es una condición poco frecuente, con manifestaciones clínicas muy inespecíficas, mecanismos fisiopatológicos desconocidos y de difícil manejo, con consecuencias potencialmente mortales. Por esta razón, debe haber un alto grado de sospecha por parte del cirujano para llegar al diagnóstico.

No existen en la literatura médica estudios en los que se comparen los métodos diagnósticos o terapéuticos para esta entidad, por lo cual, hasta el día de hoy, existen grandes controversias al respecto. Es necesario que se lleven a cabo nuevas investigaciones en este campo.

Conflicto de interés. Los autores declaramos no tener conflictos de interés.

Fuentes de financiación. Este estudio fue autofinanciado.

\section{Referencias}

I. Pareja-Pineda JI. Calcificación del ligamento estilohioideo y apófisis estiloides elongada: hallazgo durante necropsia médico-legal por muerte súbita. Colombia Forense. 2015;2:IOI-6. doi: I0.I6925/cf.v3iI.II76

2. Emary PC, Dornink M, Taylor JA. A rare case of Eagle syndrome and diffuse idiopathic skeletal hyper- ostosis in the cervical spine. J Can Chiropr Assoc. 2017;61:167-70.

3. Smoot TW, Taha A, Tarlov N, Riebe B. Eagle syndrome: A case report of stylocarotid syndrome with internal carotid artery dissection. Interv Neuroradiol. 20I7;23:433-6. doi: I0.II77/I5910I9917706050

4. Kamal A, Nazir R, Usman M, Salam BU, Sana F. Eagle syndrome; radiological evaluation and management. J Pak Med Assoc. 20I4;64:1315-7.

5. Skrzat J, Mróz I, Walocha J, Zawiliński J, Jaworek JK. Bilateral ossification of the stylohyoid ligament. Folia Morphol (Warsz). 2007;66:203-6.

6. Aldelaimi TN, Boskani SW, Ali SM, Mahmud PK. Eagle syndrome: An unusual cause limited mouth opening and surgical management. J Craniofac Surg. 2017;28:e2I4-6. doi: I0.IO97/SCS.ooooooooooo03399

7. Lei J, Yap AU, Zhang Y, Fu KY. Unilateral complete articulated ossification and aberrant thickening of the stylohyoid chain. J Oral Sci. 2017;59:157-I60. doi: 10.2334/ josnusd.I6-0383

8. Chaves H, Costa F, Cavalcante D, Ribeiro T, Gondim D. Asymptomatic bilateral elongated and mineralized stylohyoid complex: Report of one case. Rev Med Chil. 2013;I4I:793-6. doi: 10.4067/Soo34-98872013000600oI5

9. Gárriz-Luis M, Irimia P, Alcalde JM, Domínguez P, Narbona J. Stylohyoid complex (Eagle) syndrome starting in a 9-year-old boy. Neuropediatrics. 20I7;48:53-56. doi: I0.I055/s-0036-I597272

Io. Gupta A, Aggrawal A, Setia P. A rare fatality due to calcified stylohyoid ligament (Eagle syndrome). Med Leg J. 20I7;85:IO3-IO4. doi: IO.II77/00258I72I7695139.

II. Jadhav SD, Margum SR. Ossification of stylohyoid ligament and its clinical significance: Case report. Asian J Biomed and Pharm Scien. 2014;4:24-26. doi: I0.15272/ ajbps.v4i33.508 